\title{
Paecilomyces lilacinus causing debilitating sinusitis in an immunocompetent patient: a case report
}

\author{
Gentle Wong ${ }^{1 *}$, Robert Nash ${ }^{1}$, Kushal Barai ${ }^{1}$, Raksha Rathod ${ }^{2}$ and Arvind Singh ${ }^{1}$
}

\begin{abstract}
Introduction: Since the discovery of the first documented case of Paecilomyces in 1963, only five cases of Paecilomyces sinusitis have been described to date and all of them have predisposing factors such as immunocompromised status or prior nasal surgery. We present the first case of Paecilomyces lilacinus sinusitis in a fit young woman with no identified predisposing factors. To the best of our knowledge, this is the first known case in the UK and in Europe.

Case presentation: A 20-year-old Iraqi woman who has lived in the UK for the past five years presented with rhinorrhea, hyposmia, and nasal obstruction. She was previously fit and well and had no significant medical history. Imaging revealed a fungal infection that was eventually revealed on cytological examination to be $P$. lilacinus.

Conclusions: $P$. lilacinus is both a difficult and important organism to identify because it has intrinsic anti-fungal resistance. In our case, the infection was severe and recurrent, and the organism demonstrated resistance to common oral anti-fungal agents. There was a delay in its diagnosis, owing to its similarity in appearance to Penicillium and a difficulty in distinguishing between the two without specialized knowledge of fungal taxonomy. In the field of otolaryngology, Paecilomyces is relatively unknown. Our intention is to raise awareness of this organism as well as to describe the challenges in its management.
\end{abstract}

\section{Introduction}

Fungal sinusitis is a common infection and its prevalence has increased significantly in the past 30 years [1]. This trend could be attributed to modern immunosuppressive agents, overuse of antibiotics, and increased public awareness. Generally speaking, fungal sinusitis can be classified as invasive - determined by the presence of fungal hyphae within the mucosa, submucosa, bone, or blood vessels of the paranasal sinuses - or noninvasive. Species of Aspergillus are the most common agents in fungal sinusitis and the next most common are a vast array of fungi, including Candida spp., Cryptococcus neoformans, Bipolaris, and zygomycetes. Effective management depends on the type of sinusitis as well as the causative fungus/fungi and can range from aggressive surgical debridement and systemic anti-fungal therapy (as in most cases of acute/chronic invasive fungal sinusitis) to surgical removal of the fungal source

\footnotetext{
* Correspondence: gentle.wong@doctors.org.uk

'Department of Otolaryngology, Northwick Park Hospital, Middlesex, HA1 $3 \mathrm{UJ}, \mathrm{UK}$

Full list of author information is available at the end of the article
}

and long-term use of topical nasal steroids (in cases of allergic fungal sinusitis). Occasionally, unusual organisms are detected and these may have implications for successful eradication and treatment.

Paecilomyces lilacinus is an opportunistic fungus commonly found in the soil but is seldom pathogenic for humans. Only five cases of a fungal sinusitis caused by P. lilacinus have been documented [2-6], and all five patients were associated with either impaired host defenses or prior surgical procedures. Here, we report the first case of $P$. lilacinus fungal sinusitis in the UK and Europe and briefly review the literature on Paecilomyces infections.

\section{Case presentation}

A 20-year-old Iraqi woman who has lived in the UK for the past five years presented with rhinorrhea, hyposmia, and nasal obstruction. A computed tomography scan of her sinuses revealed a left-sided posterior nasal polyp associated with a sphenoidal mucocele. On both T1and T2-weighted images, magnetic resonance imaging (MRI) of the sinuses demonstrated hypointensity in
C Biomed Central 


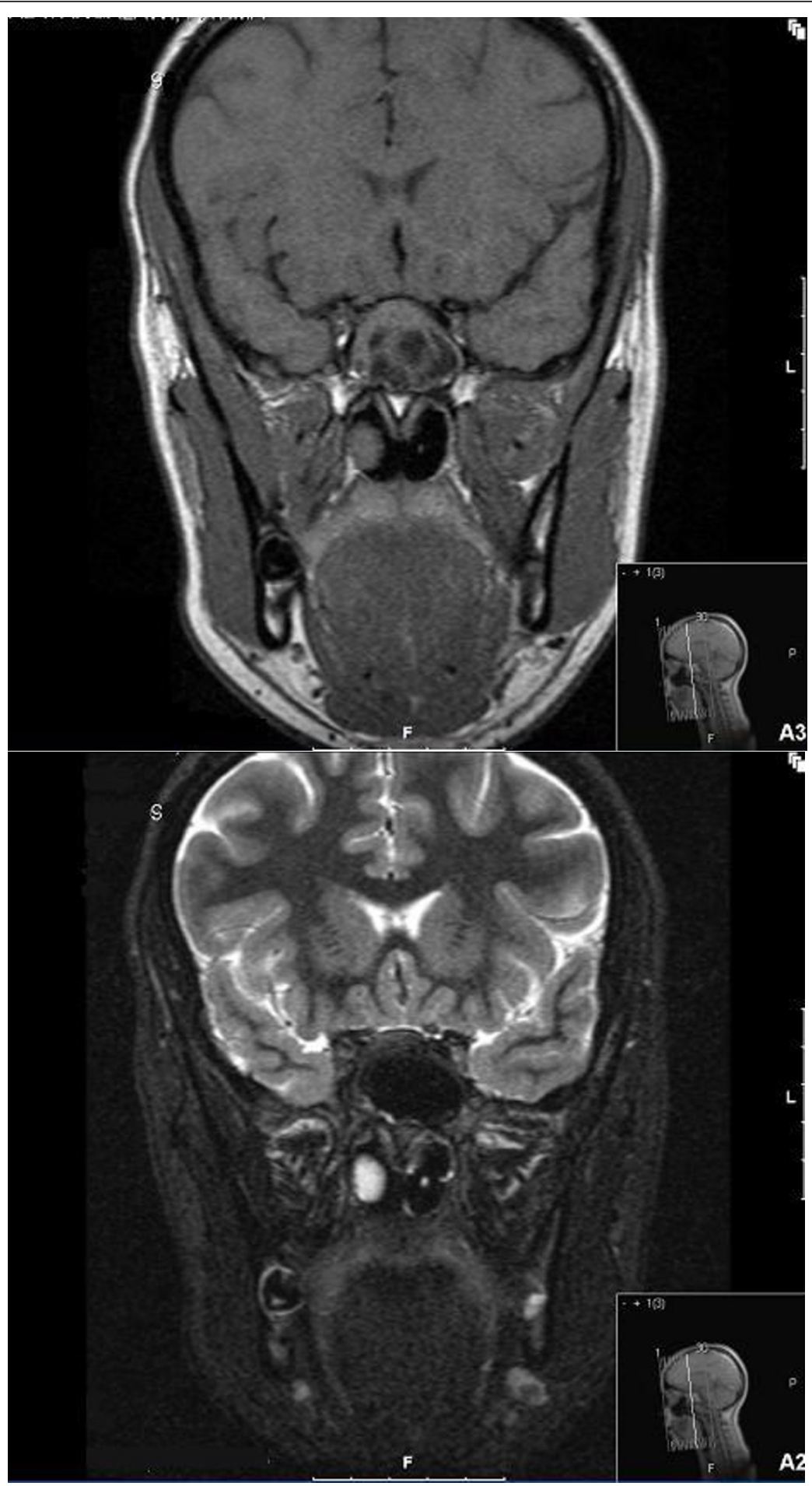

Figure 1 Magnetic resonance images of sinuses demonstrate a fungal infection. Coronal T1-weighted (above) and T2-weighted (below) images demonstrate enlargement of the left sphenoid sinus. The posterior aspect of the left nasal polyp and the contents of the large sphenoid sinus are of low T2 and short inversion time inversion recovery (STIR) signal intensity, indicating the presence of coexisting fungal infection. 

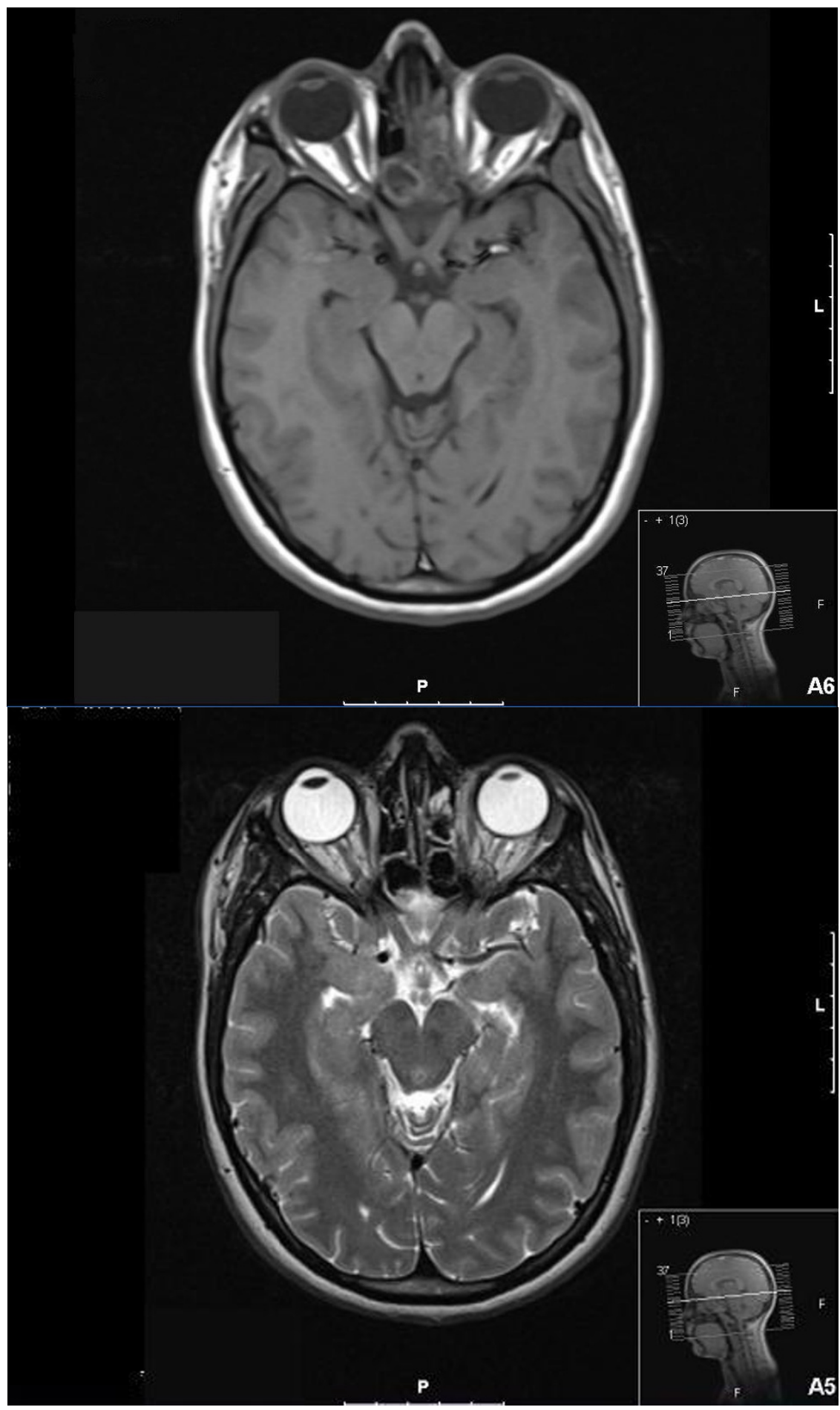

Figure 2 Magnetic resonance images of sinuses demonstrate a recurrence of the fungal infection. Axial T1-weighted and T2-weighted images show opacification of the sphenoid sinus on the left with signal characteristics of fungal infection. The infection extends into the left posterior and anterior ethmoidal air cells. 


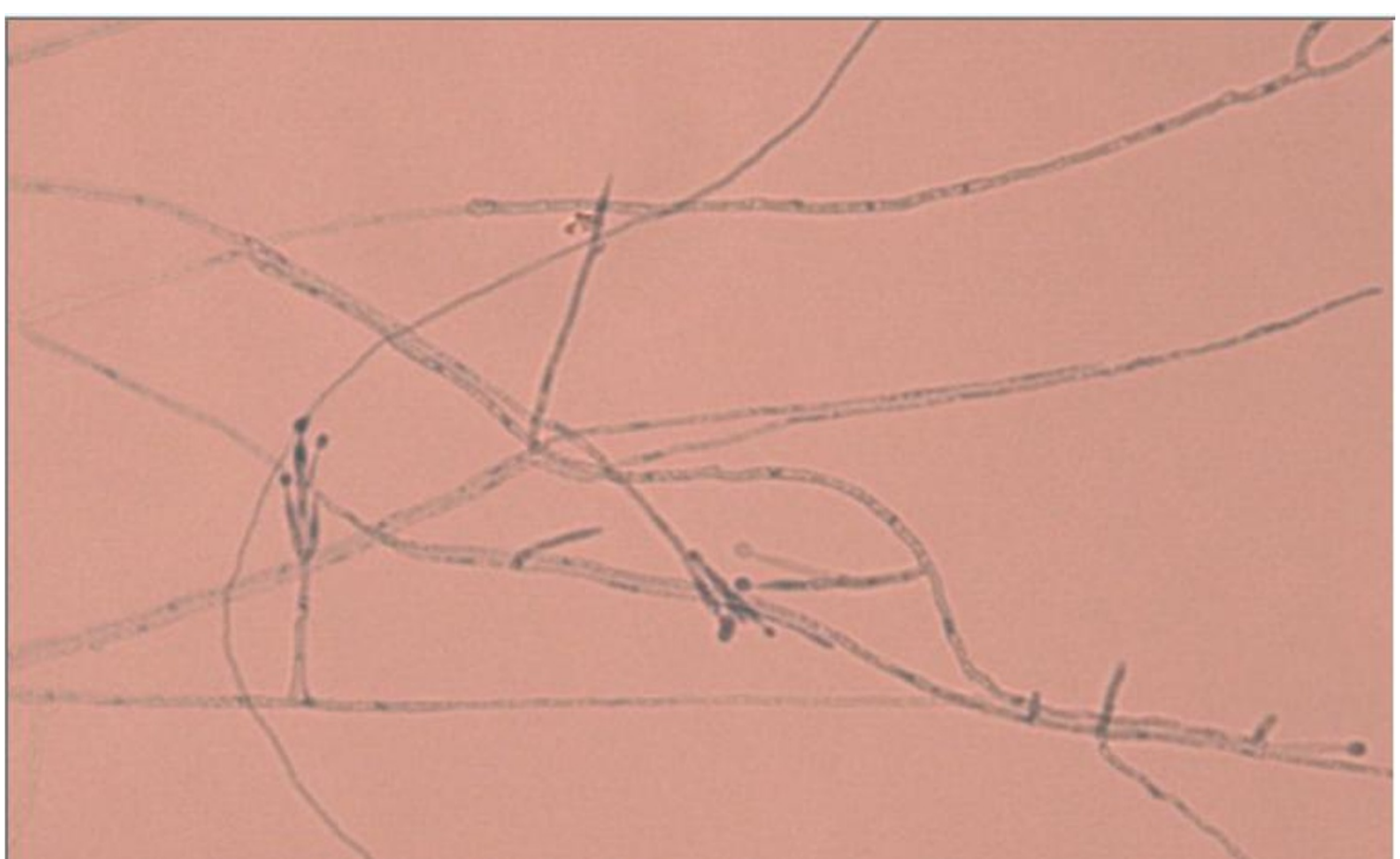

Figure 3 Septate hyaline hyphae, conidiophores, phialides, conidia, and chlamydospores. Conidiophores ( 3 to $4 \mu \mathrm{m}$ wide and 400 to 600 $\mu \mathrm{m}$ long) are branched and carry the phialides at their tips. The phialides are swollen at their bases and taper toward their apices.

keeping with fungal infection (Figure 1). She underwent endoscopic sinus surgery and clearance of the collection. A microbiology specimen isolated Staphylococcus aureus. Histology revealed numerous fungal hyphae.

After the operation, her symptoms initially improved but subsequently recurred despite topical steroid and saline douches. She continued to have headaches and nasal discharge. Repeat MRI described a more extensive infection, extending into the ethmoidal air cells (Figure 2). During revision surgery, offensive florid fungal sinusitis was extensively cleared. Cytology identified P. lilacinus (Figure 3). She made a good post-operative recovery.

\section{Discussion}

A MEDLINE search for all cases of Paecilomyces infections involving paranasal sinuses was performed to assess frequency, clinical presentation, and treatment (Table 1). We found that all five patients who have contracted the infection were associated with either impaired host defenses or previous nasal procedures.

In all parts of the body, there have been only four published reports of a cytologically confirmed infection due to P. lilacinus in a patient without predisposing factors [7-10]. Long-term treatment with itraconazole led to resolution of the ocular and cutaneous infections $[7,9]$.

The two major pathogenic species of genus Paecilomyces are P. lilacinus and $P$. variotti. P. lilacinus is documented as the more pathogenic agent while also being more resistant to anti-fungal therapy, notably amphotericin B and flucytosine [11,12]. This underscores the need to accurately identify the correct species. Worryingly, our specimen was also resistant to

Table 1 Paecilomyces paranasal sinus infections

\begin{tabular}{llcccll}
\hline Case & Year & Reference & Country & Age in years/Gender & Predisposing factors & Treatment \\
\hline 1 & 1980 & {$[2]$} & USA & $47 /$ Female & Previous nasoantrostomy & Surgery \\
\hline 2 & 1982 & {$[3]$} & USA & $47 /$ Female & Previous nasoantrostomy & Surgery \\
\hline 3 & 1996 & {$[4]$} & USA & $22 / F e m a l e$ & Myeloid leukemia & AMB, 5FC, and ITZ \\
\hline 4 & 1997 & {$[5]$} & USA & $57 /$ Female & Diabetes mellitus & Surgery, AMB, and ITZ \\
\hline 5 & 2000 & {$[6]$} & India & $8 /$ Male & Previous nasoantrostomy & Surgery and ITZ \\
\hline
\end{tabular}

5FC: 5-flucytosine; AMB: amphotericin B; ITZ: itraconazole. 
itraconazole, which was the successful anti-fungal agent in previous cases. Our specimen was sensitive to caspofungin and voriconazole, both requiring parenteral administration in our hospital.

Furthermore, in our case, morphological identification using standard methods was inconclusive. The sample was inoculated onto sabouraud dextrose agar + chloramphenicol and sabouraud dextrose agar + chloramphenicol + actidione. The plates were incubated at $37^{\circ} \mathrm{C}$ and $30^{\circ} \mathrm{C}$ for 10 days. Growth was observed at $30^{\circ} \mathrm{C}$ on both plates at days three to five, and eventually the isolate grew at $37^{\circ} \mathrm{C}$ after further incubation. The cultures grew as a white mold that failed to pigment initially, but after several weeks of further incubation, the culture became lilac. This explains the difficulty in initial identification. Ultimately, a definitive identification was established through molecular analysis at the Mycological Reference Laboratory in Bristol, UK.

The problem with identification has been reported before, as micro-morphological analysis reveals that the reproductive structures of Paecilomyces and Penicillium are similar in appearance and difficult to distinguish without specialized optics and measuring devices not available in most clinical laboratories. Gottlieb and Atkins [9] showed that the internal transcribed spacer (ITS) regions within the recombinant deoxyribonucleic acid (rDNA) complex were effective molecular targets for the identification of Paecilomyces. The difficulty in isolating the organism suggests that, in some cases, sinusitis could be caused by P. lilacinus but attributed to other microorganisms, as occurred in our case, in which S. Aureus was initially identified as the causative agent, even though hyphae were also present.

\section{Conclusions}

We report the first case of $P$. lilacinus sinusitis in an immunocompetent adult who had had no prior nasal surgery. Incidentally, this is also the first case in the UK and in Europe. P. lilacinus may cause aggressive infections and often demonstrates multi-drug resistance. In our case, these drugs included itraconazole, fluconazole, amphotericin, and flucytosine. This is therefore an important new differential diagnosis to consider because of the organism's potential multi-anti-fungal resistance, which can complicate medical management, and the difficulty in its identification, which can lead to delays in initiating treatment.

\section{Consent}

Written informed consent was obtained from the patient for publication of this case report and accompanying images. A copy of the written consent is available for review by the Editor-in-Chief of this journal.

\section{Abbreviations}

MRI: magnetic resonance imaging.

\section{Acknowledgements}

The authors thank Susan Howell for photographing the specimen slides and Guduru Gopal Rao for his invaluable microbiological advice.

\section{Author details}

Department of Otolaryngology, Northwick Park Hospital, Middlesex, HA1 3UJ, UK. ²Department of Microbiology, Northwick Park Hospital, Middlesex, HA1 3UJ, UK.

\section{Authors' contributions}

GW was the chief author of the manuscript. RN was involved in revising the manuscript critically for important intellectual content. KB was involved in the patient's care and the acquisition of the imaging data. He also obtained the patient's consent for publication of this case report and accompanying images. RR was the specialist biomedical scientist who examined and identified the organism. AS was the consultant who supervised the case report. All authors read and approved the final manuscript.

\section{Competing interests}

The authors declare that they have no competing interests.

Received: 28 July 2011 Accepted: 26 March 2012

Published: 26 March 2012

\section{References}

1. American Academy of Otolaryngology - Head and Neck Surgery: Fungal sinusitis.[http://www.entnet.org/Healthlnformation/Fungal-Sinusitis.cfm].

2. Rockhill RC, Klein MD: Paecilomyces lilacinus as the cause of chronic maxillary sinusitis. J Clin Microbiol 1980, 11:737-739.

3. Rowley SD, Strom CG: Paecilomyces fungus infection of the maxillary sinus. Laryngoscope 1982, 92:332-334.

4. Guclap R, Carlisle P, Gialanella P, Mitsudo S, McKitrick J, Dutcher J: Paecilomyces sinusitis in an immunocompromised adult patient: case report and review. Clin Infect Dis 1996, 23:391-393.

5. Van Shoonveld T, Freifeld A, Lesiak B, Kalil A, Sutton DA, Iwen PC: Paecilomyces lilacinus infection in a liver transplant patient: case report and review of literature. Transpl Infect Dis 2008, 10:117-122.

6. Nayak DR, Balakrishnan R, Nainani S, Siddique S: Paecilomyces fungus infection of the paranasal sinuses. Int J Pediatr Otorhinolaryngol 2000 52:183-187.

7. Gutiérrez-Rodero F, Moragón M, Ortiz de la Tabla V, Mayol MJ, Martin C: Cutaneous hyalohyphomycosis caused by $P$. lilacinus in an immunocompetent host successfully treated with itraconazole: case report and review. Eur J Clin Microbiol Infect Dis 1999, 18:814-818.

8. Westenfeld F, Alston WK, Winn WC: Complicated soft tissue infection with prepatellar bursitis caused by Paecilomyces lilacinus in an immunocompetent host: case report and review. J Clin Microbiol 1996, 34:1559-1562.

9. Gottlieb T, Atkins BL: Case report. Successful treatment of cutaneous Paecilomyces lilacinus infection with oral itraconazole in an immunecompetent host. Mycoses 2001, 44:513-515.

10. Gutierrez F, Masia M, Ramos J, Elia M, Mellado E, Cuenca-Estrella M: Pulmonary mycetoma caused by an atypical isolate of Paecilomyces species in an immunocompetent individual: case report and literature review of Paecilomyces lung infections. Eur J Clin Microbiol Infect Dis 2005, 24:607-611.

11. Houbraken J, Verweij PE, Rijs AJ, Borman AM, Samson RA: Identification of P. variotti in clinical samples and settings. J Clin Microbiol 2010, 48:82754-82761.

12. Pastor FJ, Guarro J: Clinical manifestations, treatment and outcome of $P$. lilacinus infections. Clin Microbiol Infect 2006, 12:948-960.

doi:10.1186/1752-1947-6-86

Cite this article as: Wong et al:: Paecilomyces lilacinus causing

debilitating sinusitis in an immunocompetent patient: a case report. Journal of Medical Case Reports 2012 6:86. 